\title{
Automating Collection Development of Non-Latin script Materials with Macros
}

\author{
Adam H. Lisbon \\ University of Colorado Boulder \\ Assistant Professor \\ Japanese \& Korean Studies Librarian \\ University Libraries \\ 184 UCB \\ Boulder, CO 80309-0184 \\ adam.lisbon@colorado.edu \\ 303-492-8822
}




\section{Automating Collection Development of Non-Latin Script Materials with Macros}

\section{Abstract}

Non-Latin scripts pose issues of labor, language fluency, and accuracy for libraries. WorldCat.org already provides multilingual data on many of the world's non-English monographs. With a macro designed to automatically search WorldCat.org it is possible to create lists of materials that faculty and students need for their research, so long as they can provide an ISBN. This is all accomplished with minimal coding knowledge. Compared to manually copying and pasting, this process is $9 x$ faster. The time saved allows librarians to concentrate on complex acquisitions, and other aspects of their professional life such as instruction, outreach, research, and service.

\section{Introduction}

Automating workflows allows librarians to focus on complex tasks that computers cannot solve. However, automation implies needing to learn to code. This is not always the case, many workflows can be automated by recording the key strokes done to complete a task. This type of automation is known as a macro. Macro recording programs allow librarians without a strong background in coding to automate their workflows. This article shows how a macro was developed to solve a complex problem with non-English non-Latin script materials that can be employed at any institution.

Libraries often collect materials in a variety of languages, some posing greater challenges than others. For those languages that do not use the Latin-based alphabetic scripts, it may not be possible to read or translate without someone fluent in the language on staff at the library. Many librarians, from public services to acquisitions and cataloging, can navigate 
through a Romance-language item. However, when materials needed for the collection are in a language like Japanese, librarians may find they cannot read the language at all.

Fortunately, WorldCat.org often provides the vernacular script as well as a Romanized transliteration. Depending on workflows in an institution, there may be a subject specialist librarian with a geographic focus who can read the language, make selections, and pass that information on to an acquisitions unit to prepare purchase orders, as is the case at the University of Colorado Boulder. However, preparing such purchase lists for readability can be time consuming and tedious.

In this article, a process for automating collection development of non-Latin script materials is presented. With a custom macro-enabled Excel worksheet and the macro scripting program AutoHotKey it becomes possible to quickly prepare lists of materials in a multitude of languages to be used across the library by those who may not possess language expertise. $\underline{A}$ LibGuide (Lisbon, 2017) was co-created alongside this article for those interested in implementation. The macro takes a time consuming process, potentially hours of work, and reduces it to minutes.

\section{Literature Review}

Articles on collection development for materials in non-Latin scripts need to be considered language by language. Focusing only on Chinese, Japanese, and Korean (CJK), the articles that emerge are only summaries of collections held in the Western world, and how they were procured. Ward (2009) analyzes workflows for foreign language materials at the Rutgers University Libraries, noting that CJK languages create the most challenging set of variables to 
acquisition workflows. Other articles are merely announcements of major acquisitions. There is a gap in the literature on automating collection development for non-Latin scripts.

Macros are not unknown to libraries however, and go at least as far back as Chroust's (1993) article on "cleaning up" screens downloaded from FirstSearch to be more human readable in Word Perfect. This theme of "cleaning up" data is common in the few articles available. Identifying individual tasks for automation, as well as summaries of macro tools and how to start using them are also available (Ouderkirk, 1999).

Bregman and Chan (2001) take a holistic approach to library automation with macros. The techniques described are out of date, but the organizational big-picture approach highlights the importance of fitting automation into a larger context. Macro implementation has been considered across many library processes. Yoose (2012) suggests diagraming workflows across technical services units to get the most out of automation, with screencasts to show how to implement macros.

Gutekanst (2001) takes a different approach, tackling the issue of overdue ILL books: providing the macro code to solve the problem. Today such information would be available through sites like Stack Overflow (2017).

While early macro creation programs were created as proprietary software, open source programs like AutoHotKey were introduced to the library community by Jermey (2005). AutoHotKey is a macro scripting program able to work across multiple programs on a PC. It is available as open source software and designed to be easy to learn. Sandberg (2014) takes a similar hand-holding approach to introducing OCLC Macro Language (OLM). 
The potential for automation has not escaped the attention of the East Asian studies librarian community. The Committee on Korean Materials created the K-Romanizer, a project chaired by Hyoungbae Lee. The K-Romanizer program was built with Autolt, an open access program for creating macros. It automates transliterating Korean hangul and hanja into ALA-LC compliant Romanization. For those in East Asian librarianship, and indeed in any discipline focusing on regions that do not use a Latin-based alphabetic script, the workflow processes are inherently more complex. Romanizing by hand while cataloging is tedious and prone to typos, but an automated process can potentially reduce these issues. This does not mean that automation is not without its problems. As Blansit (2008) points out, there are hazards associated with automation and programming in general that any would-be programmer has to think about carefully.

Chen and Chen (2015) observed that most East Asian studies librarians "hold more job responsibilities, such as acquisitions and cataloging" in addition to their subject librarian duties. Therefore, East Asian studies librarians need automation processes to better balance their professional duties. Nearly all the literature points to saving time, less error prone output, and even the ergonomic benefits of not doing repetitive motions (Bregman and Chan 2001), as reasons for adopting macros and automation in general. Further, it allows librarians to focus their time on complex and sophisticated work that automation does not lend itself to.

Finally, it is worth an anecdotal mention that articles, presentations, and discussions about automation occur throughout the library world outside the peer-review process. Barnes (1997) discussed how to automate library budget tracking within Excel. In current contexts this 
includes Yoose's 2012 blog posts. Message boards, Reddit threads, Q\&A support threads for automation products all act as diverse sources for discussions on automation.

\section{Issues in Collection Development for Non-Latin Scripts}

Libraries and vendors have well established means of collecting and acquiring English language materials. Libraries use many automated tools and services provided by monographic vendors, as well as the benefit of communicating in the same native language with similar culturally constructed expectations for conducting business. For non-English Latin-script languages, such as Spanish or German, acquisitions staff may not be able to translate the meaning of the language, but the script is still readable, enabling the identification of titles, authors, and other bibliographic information needed to prepare purchase orders and communicate with vendors. However, for non-Latin scripts it becomes unrealistic for libraries of all sizes to maintain a linguistically diverse staff that can read the world's non-Latin script languages: from other alphabetic languages like Armenian (hujtntul) and Cyrillic (Кириллица), to character-based scripts like Chinese (中文) and Japanese (日本語). Adding to these challenges, most of these scripts have "classical" variants and other historical changes. Languages spoken across national boundaries may be written in different scripts. For instance, Azerbaijani is written in Cyrillic if spoken in Russia, Latin letters if spoken in Azerbaijan, or Perso-Arabic if spoken in Iran.

Then there is the technical challenge of setting up operating systems to type in nonLatin languages with either Microsoft's IME (Input Method Editor) or Apple's Input Sources, introducing another layer of challenges. There are also barriers to communicating: how do you 
accurately convey to both your acquisitions team and vendors the materials you are seeking?

Submitting lists of requested materials only in the non-Latin vernacular script means colleagues cannot read purchase orders. However, if the materials request list only includes the Romanization, vendors may not know what materials are being requested, especially if the Romanization system in the English-speaking world is different from the one used in the country of origin. The safest approach is to do both: provide titles, authors, and publishers in the vernacular and again in transliteration. This is time consuming work that takes away from other responsibilities: instruction, outreach, reference and research consultations, committee work, campus events, professional development, or time that could be spent working on more complex collection development work that automation could never handle. In the author's own experience, preparing a material request for acquisitions that has an accurate and complete WorldCat record takes at least 6-7 minutes when done manually.

\section{Macros and Workflows}

The workflow presented in this article deals with one specific part of collection development: preparing material requests from faculty and students for purchase by acquisitions staff who do not possess reading knowledge of non-Latin script languages. The librarian acts as the liaison between the two groups, assuring that the materials needed are accurately conveyed in a format that is easy for acquisitions staff to work with. The process here takes a set of ISBNs provided by users and creates a spreadsheet with bilingual bibliographic data that can be used by acquisitions staff to communicate accurate order information with vendors. 
Because macros are inherently recursive (Bregman and Chan 2001), performing the exact same set of steps in a static environment, they need to be narrow in scope. ISBNs that users provide are collected through a GoogleForm which is designed to be as simple and user friendly as possible (see Appendix A for a screenshot). It only requires a control number, typically an ISBN. If there is no ISBN, users can still provide a title, author, or other bibliographic information. Creating a standard way to receive item requests, especially one that emphasizes providing an ISBN, can save time and labor. Focusing on providing the ISBN saves time for both the librarian and the user who needs the item. The librarian receives a user-generated list of ISBNs, while the user only needs to provide one piece of data instead of providing various bibliographic data in the vernacular and transliteration.

For items requested by Japanese studies faculty and graduate students at the University of Colorado Boulder, $80 \%-90 \%$ of Japanese books requested are already on WorldCat. The macro can retrieve vernacular and transliterated bibliographic information quickly, swapping back and forth from a Google Chrome browser window to Excel. Older items without ISBNs need to be searched manually by someone with vernacular language skills such as the subject specialist. Items that are not found in WorldCat usually require specialized knowledge to locate. The final spreadsheet is reviewed by the subject specialist or someone with vernacular language skills for occasional errors. The spreadsheet is submitted to acquisitions where the details are used to create purchase orders and communicate with vendors.

\section{Making the Macro}

The acquisitions macro is actually two separate macros. The first one is the "data collection" macro. Working at the operating system level across multiple programs, it collects 
bibliographic data from WorldCat.org and populates an Excel spreadsheet. The macro itself is just a complex set of keystrokes (see Appendix C for the full keystroke command of this process). When this series of precise keyboard commands is run as a macro, it looks at an ISBN in Excel, copies it, and uses it to run a search on WorldCat.org and extract essential bibliographic data that an acquisitions department needs to place a purchase order for nonLatin script materials: author, title, and publication data both in the vernacular and Romanized scripts.

These keyboard strokes are recorded with the program Pulover's Macro Creator (Pulover). Using Pulover requires no knowledge of scripting or programming. Pulover's main purpose is only to record the macro, not run it. Pulover can also record mouse clicks but they were avoided entirely because this macro was designed to be used on multiple computers, which often have monitors with different screen resolutions. This means that the XY coordinates of the mouse clicks change system to system and would introduce a point of failure because the $X Y$ coordinates on two different screens would not "click" in the same location.

Pulover can export the recording as an AutoHotKey (AHK) file. AHK is another openaccess program for creating macros, but requires programming knowledge to create them. Using Pulover circumvents the need to learn to program in AHK, while still creating an executable macro that can be activated anytime data needs to be collected.

To run the "data collection" macro, it needs to be activated first. This is very much like opening a program. When the macro is "open," it will only run when a certain combination of keys are typed. For the macro described here it is ctrl+shift+0 (zero). It can also be stopped with the F8 key. The other two macros in Excel are more straight forward. The custom spreadsheet 
that works together with AHK has two buttons built into it. Each one runs a separate macro to help clean up the data.

This Pulover/AHK data collection macro was designed to be used specifically in Google Chrome. It was tested in Firefox, but because of slight variations in how Chrome and Firefox function, it cannot work in the latter. Chrome was specifically chosen because it is relatively easy to create multiple profiles (Google 2017). Users can create a separate profile for collection development where the custom Adblock Plus filters (see Appendix B) are always active.

The second macro cleans the data that was pulled from WorldCat.org into Excel by the data collection macro created with Pulover/AHK. This "data cleaning" macro was made entirely in Excel through the "Developer" tab, which is hidden by default, but can be revealed by changing settings in Excel's options menu. Excel, like Pulover, features a recorder that can generate a macro with no programming knowledge. Unlike Pulover, mouse clicks can be used to record macros in Excel because the cells that are activated are recorded, not the "click."

The "data cleaning" macro performs three important tasks. First, it normalizes the ISBNs that users submit, removing spaces, hyphens, and ensuring the cells in the ISBN column are formatted correctly. Second, it removes all formatting from the data copied over from Worldcat.org. Finally, through a series of carefully sequenced "find and replace" commands, it cuts out the "garbage" html from Worldcat.org and leaves behind only the human readable information: the title, author, etc., of the work in both vernacular Japanese and transliterated Latin-letters. Figure 1 diagrams out how these find and replace commands work for the title of a Japanese book: 


\section{Figure 1: Find and replace commands to remove Wordcat.org HTML from Japanese titles}

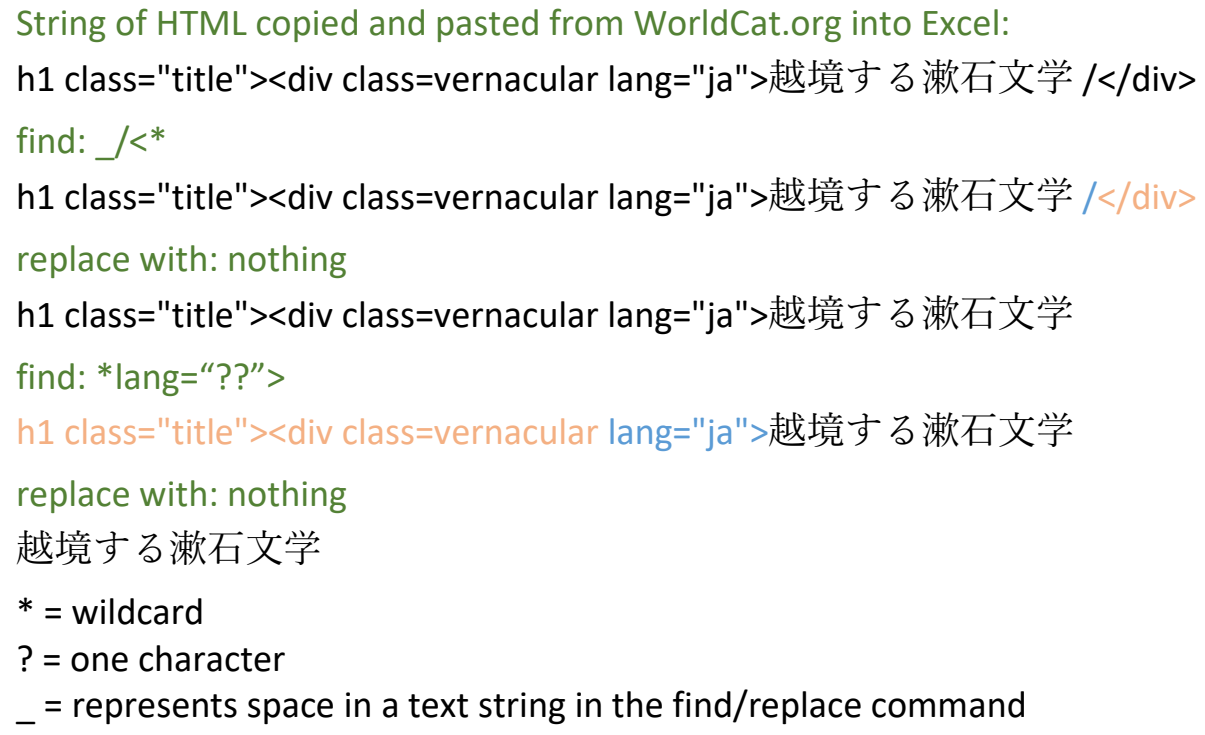

\section{Creating Macro Friendly Environments}

Macros rely on consistent environments to run optimally, and the "data collection" macro needs to hit the tab key exactly two times to move the cursor from WorldCat.org search bar to the first result after a search results page loads. WorldCat.org presents a challenge because it provides contextual information with its search results. For example, searching the ISBN 9784062190657 gives two results for the same book (OCLC 2017) and WorldCat generates a facets menu on the left. This is problematic because the facets menu increases the number of tabs needed to focus on the link to the first search result and thus becomes a "point of failure" for the macro.

To fix this, another piece of software is used to make WorldCat render in a minimalistic way. The browser plugin Adblock Plus is a common open-source plugin that suppresses obtrusive advertising on websites. This plugin can also be used to suppress any webpage element. With a custom set of filters in Adblock Plus (see Appendix B), the WorldCat homepage, 
search results, and item record page only load the web elements needed for the AHK macro to run. Figure 2 shows comparative screen captures of the default WorldCat pages and the same pages with the custom AdBlock Plus filters active.

Figure 2: Unsuppressed and Adblock Plus filtered WorldCat.org pages:
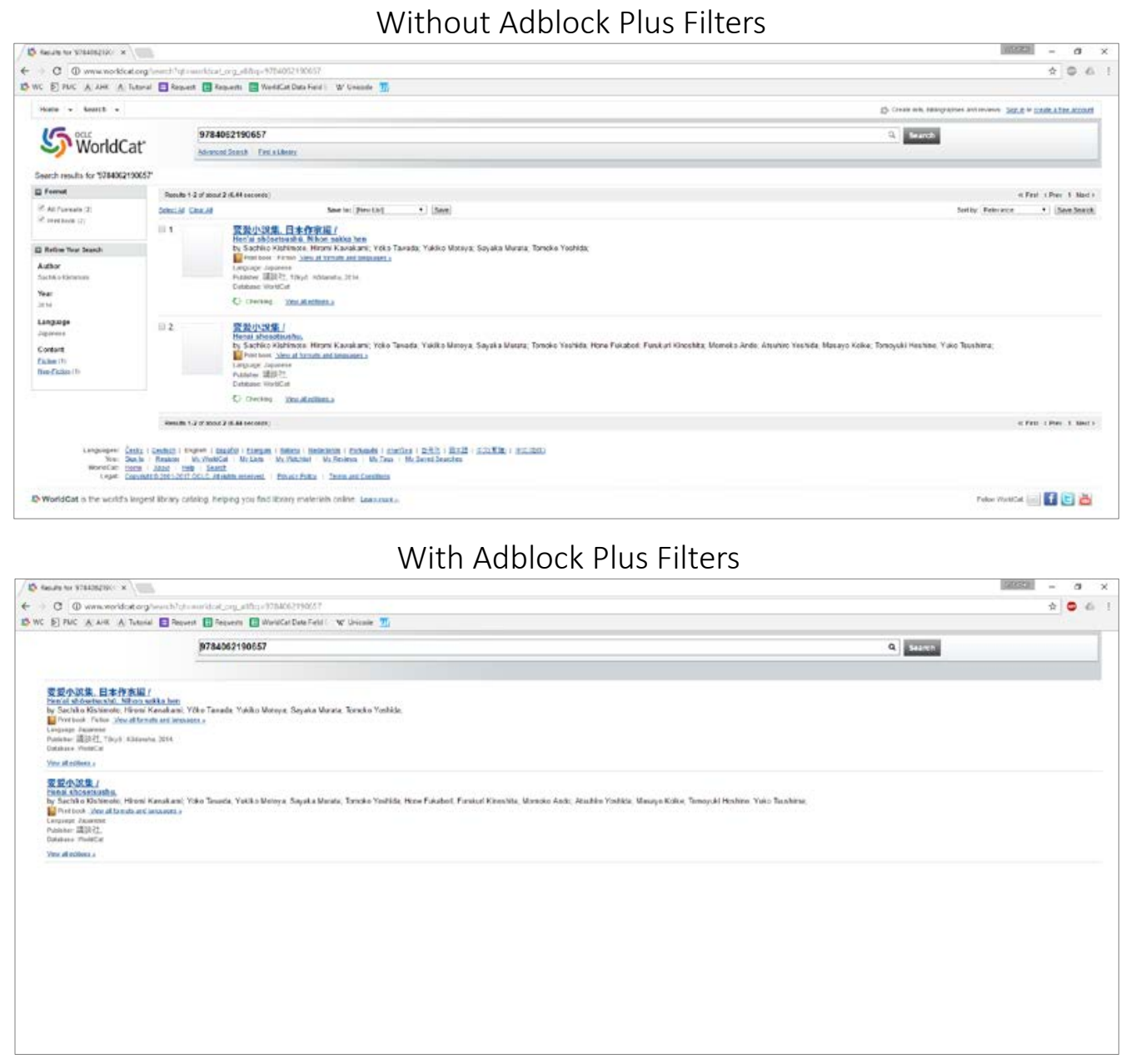

These "stabilized" versions of WorldCat.org allow the macro to be presented with a consistent environment in order to extract the necessary vernacular and transliterated data. By suppressing responsive elements on WorldCat.org and making data display consistently, the macro does not encounter changing environments and will produce better results. 


\section{Non-Latin and Japanese specific optimizations}

This macro has two specific optimizations to deal with the unique needs of collecting non-Latin language materials. First, it replaces all composed letters with diacritics with their decomposed equivalents. For example, ō and ō may look the same, but to the computer they are two different values. The first ō is a single character with a hexadecimal value of 0X14D. The second $\overline{0}$ is actually two separate values: $\mathrm{o}$ and ${ }^{-}$, with hexadecimal values $0 \times 6 \mathrm{~F}$ and 00AF respectively. These values refer specifically to a Unicode code point (Unicode Inc.) The macron in the second example is a floating diacritic that computers merge with any other character, resulting in what looks like one glyph. These decomposed characters can be searched with a ctrl+f command, because the o value matches a "regular" 0.

The second optimization deals with Japanese publisher names. WorldCat records provided by Japanese institutions that do not follow the Library of Congress ALA-LC Romanization Tables (2016). This is most noticeable in publisher fields. For example the Japanese publisher Asahi Shimbun Shuppan is often written as Asahishimbunshuppan with no word parsing or capitalization. At the time of writing, the macro currently fixes this issue with 33 Japanese publishers with more fixes being added as they are discovered. Because of the modular nature of the macro, it is possible to add more lines of code to the macro each time a new issue arises.

\section{Benefits and Results of Implementation}

As the macro is currently implemented, it saves an enormous amount of time. Testing how quickly information can be copied and pasted manually from WorldCat.org to Excel, it takes 6-7 minutes per item. The macro accomplishes the same in about 40 seconds. This is $9 x$ 
faster than collecting the data manually. It also has the benefit of protecting against repetitive motion disorders.

It also frees non-cataloging staff from having to learn 19 pages of rules for specific Romanization conventions in Japanese, let alone learning how to Romanize the other 173 languages that have Romanization conventions prescribed by the American Library Association and Library of Congress.

At 6 minutes per item, the 476 requests received in fiscal year 2016-17 would have taken 47.6 hours. At $\$ 20$ per hour that would be $\$ 952$ spent on this task. In comparison the macro could have accomplished this work in approximately 5.28 hours.

\section{Points of Failure}

The macro does have some specific points of failure. The most significant is waiting for webpages to load. The macro waits 10 seconds for WorldCat.org search result pages and item record pages to load. Because the macro cannot "sense" when webpages are finished loading, it will continue with outputting keyboard commands regardless and produce "garbage data." Another point of failure is ISBNs that produce multiple results. The first item is not always the "best" choice, and may be completely wrong in some cases. The macro will only pull data for the first search result which necessitates human review of the Excel file. This typically manifests as a book in the wrong language and is easy to spot.

The final point of failure is ISBNs that produce no results in WorldCat. This is usually because the item is too new and no records have been added to WorldCat yet, or the item may be so old that it was published before Japan started using ISBNs. In these cases, the macro will run and leave an empty row in Excel. When materials are brand new, the bibliographic data can 
be obtained manually from a website like Amazon.jp or Rakuten.co.jp, though they do not provide Romanized data because they are commercial sites selling Japanese goods to the Japanese consumer market.

While these failure points do not make the macro truly $100 \%$ automated, it succeeds in over $90 \%$ of cases. This saves a substantial amount of time, allowing librarians to focus on the remaining $10 \%$. For example, in a list of 20 items, 18 of them could be processed automatically. Assuming those 18 items were processed manually at 6 minutes each, it would take 1 hour and 45 minutes to complete a list for acquisitions. With the macro it would only take 12 minutes.

The remaining two items, if not particularly rare, could be processed in approximately 12 minutes. Even with points of failure, the cost savings on time more than make up for the $10 \%$ of items that cannot be processed automatically.

\section{Making the Macro Easy to Use for All Area Studies Librarians}

Usability is always a concern when designing a tool for a large audience. There are some manual steps that must be performed for the AHK macro to run correctly. After that, users must remember the keyboard command to activate it. For the Excel macros, instead of remembering more keyboard commands, users can press a button instead (See Figure 3).

Future plans to improve usability include a LibGuide (Lisbon, 2017) clearly outlining how to work with the macros. This LibGuide will cover what software is needed to run the macros, how they work, troubleshooting, etc. 


\section{Figure 3: Usability Features}

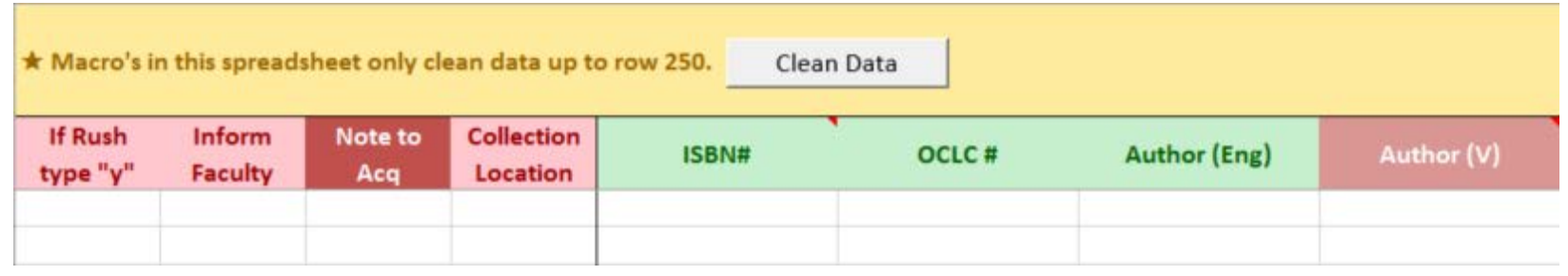

\section{Future Optimizations}

Currently, the Excel macros clean away the HTML code around the metadata for the actual author, title, etc. However, they rely on looking for the string vernacular="jp" because it is a consistent and unique text string pulled into Excel. The find and replace commands can be edited to look for vernacular="??", allowing the Excel macro to clean the data for any language, not just Japanese. Rigorous testing is needed to confirm there are no unintended side effects from altering the macro. Currently, Korean and Russian materials appear to process accurately. Arabic, Chinese, Hindi, and other non-Latin scripts need further testing.

Even during the writing of this article, the author received advice communicating with the AutoHotKey and Visual Basic communities on Reddit, and given suggestions on how to optimize the macro scripts (“Optimizing Excel Macro • R/Vba” 2017; “How to Identify Correct Program Window • R/Autohotkey" 2017). This process of optimization and improving is iterative. Even within the online discussions mentioned, new technologies were suggested that would likely provide even more stable macros. However, it also moves away from the core goal of demonstrating that automation is possible without detailed programming knowledge.

A key optimization will be an additional macro that can identify incorrect metadata brought over from WorldCat. There are a variety of ways in which this manifests. For example, Japanese WorldCat records do not follow Resource Description and Access (RDA) conventions 
used in the US. Japanese books that are part of multi-volume sets have their volume number incorporated into the title of the WorldCat record, even if it is not written in the title page. Compare OCLC\# 704165184 with OCLC\# 939215521 (See Figure 4).

Figure 4. Comparison of US and Japanese Worldcat.org records for same item

Worldcat.org Record from US Institutions $\leq<$ Return to Search Results

Write a review Rate this item:

\section{草双紙集 I}

Kusa zoshishu.

Author:

木村八重子, 宇田敏彦, 小池正胤校注木村,八重子 宇开

Worldcat.org Record from the National Diet Library of Japan $\leq<$ Return to Search Results

\section{新日本古典文学大系. 83, 草双紙集 $I$} Shin nihon koten bungaku taikei. 83, Kusazōshishū

Author:

佐竹昭広 [ほか]編; 木村八重子,宇田敏彦,小池正胤校注 $\underline{\text { Uda; }} \underline{\text { Masatane Koike }}$

\section{Future Directions}

The next step is putting the macro into the library community to test how the macro handles unforeseen scenarios. Community adoption and user experiences will create avenues for future developments and improvements, as well as the opportunity to crowdsource specialized knowledge about non-Latin script languages and how to incorporate them. To compliment this initial research, the author has developed a LibGuide dedicated to automating non-Latin collection development. 


\section{Conclusion}

Programming knowledge is of course helpful, but it is entirely possible to create macros without such skill. Since learning to code can be a time consuming process, librarians should consider the use of macro recorders. Macro recorders have their own learning curve, but allow users to become familiar with automation processes with less labor. In addition, working with this type of software may encourage librarians to start learning to program. As Hyoungbae Lee, lead creator of the K-Romanizer points out:

"IT people may be better in programming, but I believe that librarians can define better what kind of workflow can be automated in their own work. That is why I started studying macro languages..."(Lee 2017)

Macro creation is an iterative process, adding features to account for changes and improving task functionality. It allows all libraries a chance to accurately collect non-Latin language data in a systematic and accurate way. Even for larger libraries with dedicated subject-specialists in areas/languages, the time savings are valuable and allow librarians to engage in other projects like managing rare and unusual items, outreach, teaching, etc.

For technical services, the macro enables clear, effective, and accurate communication with vendors and placement of orders without having language expertise in-house. Where staffing resources are limited, technological solutions such as a macro enhance and enable accurate collection development and acquisition of materials across a range of languages, ultimately providing students and faculty with materials that might not otherwise be available. 


\section{References}

“AutoHotkey Q \& A・R/AutoHotkey." 2017. Reddit. Accessed March 22. https://www.reddit.com/r/AutoHotkey/.

Barnes, Marilyn E. 1997. “Managing with Technology: Automating Budgeting from Acquisitions." The Bottom Line 10 (2) (June): 65-73. doi:10.1108/08880459710162993. Blansit, B. Douglas. 2008. "Automating Repetitive Tasks: Getting Started with Hotkeys.” Journal of Electronic Resources in Medical Libraries 5 (2) (April): 187-198. doi:10.1080/15424060802064469.

Bregman, Alvan, and Winne S. Chan. 2001. "Customization of Library Service in a Cross-Platform Programming Environment." Information Technology \& Libraries 20 (1) (March): 21.

Chen, Yao, and Xi Chen. 2015. “Web-Based Subject Guides for East Asian Studies: Current Status, Challenges, and Recommendations." Internet Reference Services Quarterly 20 (12) (April 3): 1-17. doi:10.1080/10875301.2015.1018474.

Chroust, David Z. 1993. “Cleaning up Downloaded Screens.” Online 17 (5) (September): 44.

Google. 2017. "Share Chrome with Others or Add a Profile - Computer - Chrome Help." Chrome Help. Accessed March 30. https://support.google.com/chrome/answer/2364824?co=GENIE.Platform\%3DDesktop \&hl=en.

Gutekanst, Joe. 2001. "Macros to Track Overdue Interlibrary Loans in OCLC Passport." Journal of Interlibrary Loan, Document Delivery \& Information Supply 11 (3) (March): 65. 
“How to Identify Correct Program Window. • R/Autohotkey." 2017. Reddit. Accessed March 30. https://www.reddit.com/r/AutoHotkey/comments/620vc4/how_to_identify_correct_pr ogram_window/.

Jermey, Jonathan. 2005. "Keyboard Macros with Autohotkey." Online Currents 20 (7)

(September): 13-12.

Lee, Hyoungbae. Email. 2017. “K-Romanizer,” March 28.

Library of Congress. 2016. “ALA-LC Romanization Tables.” Library of Congress. February 24. https://www.loc.gov/catdir/cpso/roman.html.

Lisbon, Adam. 2017. “Research Guides: Automating Non-Roman Collection Development: Home." Accessed June 1. http://libguides.colorado.edu/nonRomanCDMacro/home.

OCLC. 2017. “Results for ‘9784062190657’ [WorldCat.org].” Worldcat. Accessed March 30. http://www.worldcat.org/search?q=9784062190657\&qt=owc_search.

“Optimizing Excel Macro • R/Vba.” 2017. Reddit. Accessed March 30. https://www.reddit.com/r/vba/comments/620q7z/optimizing_excel_macro/.

Ouderkirk, Jane Padham. 1999. "Technical Services Task Assignment: From Macros to Collection Management Intelligent Agents." Journal of Academic Librarianship 25 (5) (September): 397.

Sandberg, Jane. 2014. “Better Living Through Macros.” Technical Services Quarterly 31 (2) (April 3): 193-195. doi:10.1080/07317131.2014.875390.

"Stack Overflow." 2017. Accessed March 22. https://stackoverflow.com/. Ward, Judit H. 2009. “Acquisitions Globalized: The Foreign Language Acquisitions Experience in a Research Library." Library Resources \& Technical Services 53 (2) (April): 86-93. 
Yoose, Becky. 2012. "Workflow Automation in Technical Services: Part 2." ACRL TechConnect Blog. http://acrl.ala.org/techconnect/post/workflow-automation-in-technical-servicespart-2. 
Appendix A:

Materials request form for Asia related materials:

This form was created in Google Forms. It also uses a special feature to prefill the "Your Name" field. Each faculty member and graduate student receives a custom link that auto-populates their name, removing a small piece of the labor associated with requesting materials for purchase.

Figure 5. GoogleForm for collecting ISBNs

QUESTIONS RESPONSES 1,487

\section{Request Asian Studies Materials}

Order Japanese and/or Korean Studies materials for the

$\star t \star$ ONLY the ISBN is required to order a BOOK, no other information is needed.

$\star$ No fields are required, but providing an ISBN is the FASTEST way to get what you need.

$\star$ Japanese Items: try to provide AUTHOR and PLACE names in Japanese AND Rōmaji

Questions about this form? Email

Your Name:

Short answer text

Control Number:

ex: ISBN, DOI, URL, PURL, etc - $\star$ Make sure there are NO TYPOS! $\star$

Short answer text

Rush Order?

Yes (needed in less than 2-3 weeks)

Other Information (Author, Title, Series, etc.) 
Appendix B:

Adblock Plus Filters to Create Macro-Friendly WorldCat.org Pages

To implement these filters in the Chrome browser, you need Adblock Plus. In the options, go to the "options" tab and click on "Edit filters as raw text." Then you can copy and paste the filters below to alter what elements are displayed on WorldCat.

worldcat.org\#\#.ipsign

worldcat.org\#\#.qm

worldcat.org\#\#.scopesummary

worldcat.org\#\#\#util-links

worldcat.org\#\#\#util-cont

worldcat.org\#\#\#soc-cont

worldcat.org\#\#\#libspot

worldcat.org\#\#.getthis

worldcat.org\#\#.borrow.downpage

worldcat.org\#\#.buyit.downpage

worldcat.org\#\#.showMoreLessContentElement.expanded-content.showMoreLessBox-darkcontent-expanded

worldcat.org\#\#.showMoreLessControlElement.expanded-control

worldcat.org\#\#\#rev-users

worldcat.org\#\#\#add_tag_signedout

worldcat.org\#\#.soc-util-cont

worldcat.org\#\#\#div-footer

worldcat.org\#\#\#div-refinefm

worldcat.org\#\#.faceted

worldcat.org\#\#.results-actions

worldcat.org\#\#.results-sort

worldcat.org\#\#\#menubar

worldcat.org\#\#.resultsinfo

worldcat.org\#\#.coverart

worldcat.org\#\#.num

worldcat.org\#\#.showMoreLessControlElement.contracted-control

worldcat.org\#\#\#brand-cont

worldcat.org\#\#\#cover

worldcat.org\#\#.signin-no

worldcat.org\#\#\#news

worldcat.org\#\#.tout-mobi

worldcat.org\#\#\#idnetworkribbon

worldcat.org\#\#\#genresribbon

worldcat.org\#\#\#mainfeature

worldcat.org\#\#.contentrow-cont

worldcat.org\#\#\#librarian 
worldcat.org\#\#.about

worldcat.org\#\#.itemsavail

worldcat.org\#\#.yui-nav

worldcat.org\#\#.homesrchadvan

worldcat.org\#\#.illo

Appendix C:

Full List of Keystrokes for Macro to Emulate to Pull WorldCat.org Data into Excel

Macro creation requires a detailed understanding of how a goal is achieved. Writing out each keystroke is like performing an audit on a workflow, and makes it easier to prepare a macro.

\begin{tabular}{|c|c|c|}
\hline STEP & KEYSTROKE(S) & PURPOSE \\
\hline 1 & control+c & copies ISBN \\
\hline 2 & alt+tab & switch to browser \\
\hline 3 & alt $+d$ & focus on address bar \\
\hline 4 & tab & move focus \\
\hline 5 & tab & move focus to WorldCat search bar \\
\hline 6 & control+v & paste ISBN \\
\hline 7 & enter & run WorldCat search \\
\hline 8 & *wait 10 seconds* & give page time to load \\
\hline 9 & tab & move focus \\
\hline 10 & tab & move focus to first search result \\
\hline 11 & enter & activate link, load page \\
\hline 12 & *wait 10 seconds* & give page time to load \\
\hline 13 & control+u & open source code \\
\hline 14 & control+f & open browser find command \\
\hline 15 & text string "oclc:" & unique text string for OCLC\# \\
\hline 16 & esc & close search command \\
\hline 17 & control+shift+downArrow & highlight OCLC\# data \\
\hline 18 & control+c & copy OCLC\# data \\
\hline 19 & alt+tab & switch to Excel \\
\hline 20 & rightArrow & move over one cell \\
\hline 21 & control+v & paste OCLC\# data \\
\hline 22 & esc & unfocus on cell \\
\hline 23 & rightArrow & move over one cell \\
\hline 24 & alt+tab & switch to browser \\
\hline 25 & controltf & open browser find command \\
\hline 26 & text string "bib-author-cell" & unique text string for author \\
\hline 27 & esc & close search command \\
\hline 28 & control+shift+downArrow & highlight author data \\
\hline 29 & control+shift+c & copy author data \\
\hline 30 & alt+tab & switch to excel \\
\hline 31 & control+v & paste author data (romanized) \\
\hline 32 & esc & unfocus on cell \\
\hline 33 & rightArrow & mover over one \\
\hline 34 & control+v & paste author data (vernacular) \\
\hline
\end{tabular}




\begin{tabular}{|c|c|c|}
\hline 35 & esc & unfocus on cell \\
\hline 36 & rightArrow & move over one cell \\
\hline 37 & alt+tab & switch to browser \\
\hline 38 & control+f & open browser find command \\
\hline 39 & text string "h1 " & unique text string for title \\
\hline 40 & esc & close search command \\
\hline 41 & control+shift+downArrow & highlight title data \\
\hline 42 & control+shift+downArrow & highlight title data \\
\hline 43 & control+c & copy title data \\
\hline 44 & alt+tab & switch to Excel \\
\hline 45 & control+v & paste title data \\
\hline 46 & esc & unfocus on cell \\
\hline 47 & downArrow & move down one cell \\
\hline 48 & upArrow & move back up one cell \\
\hline 49 & control+x & cuts vernacular title data \\
\hline 50 & rightArrow & move over one cell \\
\hline 51 & control+v & paste vernacular title data in vernacular title column \\
\hline 52 & downArrow & move down one cell \\
\hline 53 & control+x & cut Romanized title data \\
\hline 54 & leftArrow & move one cell left \\
\hline 55 & upArrow & move up one cell back to correct line of data \\
\hline 56 & control+v & paste Romanized title data in Romanized title column \\
\hline 57 & alt+tab & switch to browser \\
\hline 58 & control+f & open browser find command \\
\hline 59 & text string "items in thi" & unique text string for series data \\
\hline 60 & esc & close search command \\
\hline 61 & control+shift+downArrow & highlight series data \\
\hline 62 & control+c & copy series data \\
\hline 63 & alt+tab & switch to excel \\
\hline 64 & rightArrow & move over one cell \\
\hline 65 & rightArrow & mover over one cell \\
\hline 66 & control+v & paste series data in series column \\
\hline 67 & esc & unfocus on cell \\
\hline 68 & rightArrow & mover over one cell \\
\hline 69 & alt+tab & switch to browser \\
\hline 70 & control+f & open browser find command \\
\hline 71 & text string "bib-publisher-cell" & unique text string for publisher data \\
\hline 72 & esc & close search command \\
\hline 73 & control+shift+downArrow & highlight publisher data \\
\hline 74 & control+c & copy publisher data \\
\hline 75 & control w & close source code tab \\
\hline 76 & alt+tab & switch to Excel \\
\hline 77 & control+v & paste publisher data \\
\hline 78 & esc & unfocus on cell \\
\hline 79 & rightArrow & move over one cell \\
\hline 80 & control+v & paste publisher data \\
\hline 81 & esc & unfocus on cell \\
\hline 82 & rightArrow & move over one cell \\
\hline
\end{tabular}




\begin{tabular}{l|ll}
$\mathbf{8 3}$ & control+v & paste publisher data \\
$\mathbf{8 4}$ & downArrow & move down one row for next ISBN \\
$\mathbf{8 5}$ & leftArrow & \\
$\mathbf{8 6}$ & leftArrow & \\
$\mathbf{8 7}$ & leftArrow & \\
$\mathbf{8 8}$ & leftArrow & \\
$\mathbf{8 9}$ & leftArrow & \\
$\mathbf{9 0}$ & leftArrow & \\
$\mathbf{9 1}$ & leftArrow & \\
$\mathbf{9 2}$ & leftArrow & \\
$\mathbf{9 3}$ & leftArrow & move back over to next ISBN \\
$\mathbf{9 4}$ & leftArrow & switch to browser \\
$\mathbf{9 5}$ & alt+tab & focus on address bar \\
$\mathbf{9 6}$ & alt+d & enter WorldCat homepage address \\
$\mathbf{9 7}$ & Text string "worldcat.org" & reloads worldcat.org for next search \\
$\mathbf{9 8}$ & enter & switch to Excel \\
$\mathbf{9 9}$ & alt+tab &
\end{tabular}

